\title{
Proposal for detection of magnetic fields through magnetostrictive perturbation of optical fibers
}

\author{
Amnon Yariv* \\ Defense Advanced Research Projects Agency, Material Research Council, Arlington, Virginia 22209 \\ Harry V. Winsor ${ }^{\dagger}$ \\ Defense Advanced Research Projects Agency, Arlington, Virginia, 22209 \\ Received November 5, 1979 \\ The possibility of detecting magnetic fields by a magnetostrictive straining of optical fibers is investigated. The \\ effect of shot noise and the limiting sensitivity are considered.
}

We analyze the possibility of detecting weak magnetic fields by using magnetostrictive perturbation of optical fibers. The basic scheme is demonstrated in Fig. 1. A low-loss optical fiber of length $L$ is sheathed in a magnetostrictive jacket that undergoes a longitudinal strain (magnetostriction) when immersed in a magnetic field. This strain affects the phase delay of a laser light beam propagating in the fiber.

Experimental data of the longitudinal strain $\epsilon_{e}$ induced by a magnetic field $H$ are shown in Fig. 2, reproduced from Ref. 1. Using this figure, we can approximate the data for nickel by

$$
\begin{gathered}
\epsilon_{3} \equiv \frac{\Delta l}{l}=\kappa H^{1 / 2}, \\
\kappa \simeq-10^{-5}(\mathrm{Oe})^{-1 / 2} .
\end{gathered}
$$

Let us assume that the total field $H$ is the sum of a bias field $H_{0}$ and a time-varying field $H_{1} \ll H_{0}$. Expanding Eq. (1) near $H_{0}$ gives [symmetry arguments dictate that in an isotropic material $\epsilon_{3}$ be a function of $\mathbf{H} \cdot \mathbf{H}$, so $\epsilon_{3}{ }^{\prime}$ is more properly expressed as $\epsilon_{3}{ }^{\prime}=\left(\kappa \mathbf{H}_{0} \cdot \mathbf{H}_{1} /\right.$ $\left.\left.\left|H_{0}\right|^{3 / 2}\right)\right]$

$$
\epsilon_{3}=\epsilon_{3}{ }^{0}+\epsilon_{3}^{\prime}=\kappa H_{0}^{1 / 2}+\frac{\kappa H_{1}}{2 H_{0}{ }^{1 / 2}},
$$

which, when we use the above value for $\kappa$ and take $H_{0}$ $=3 \mathrm{G}$, leads to

$$
\epsilon_{3}{ }^{\prime} \simeq-2.89 \times 10^{-6} H_{1}(\mathrm{G}) .
$$

In the case of the fiber geometry of Fig. 1, we assume that the cross-sectional area of the magnetostrictive jacket is much larger than that of the silica fiber. In this case we expect the strain $\epsilon_{3}$ inside the fiber to be equal to that of the jacket and thus to be given by Eq. (1). The magnetostriction is also taken to be associated with zero net-volume change, so

$$
2 \epsilon_{1}+\epsilon_{3}=0 \text {. }
$$

Here $\epsilon_{1}=\epsilon_{2}$ are the transverse $(1,2)$ diagonal strain components and $\epsilon_{3}$ is the longitudinal component. It follows that in addition to the strain $\epsilon_{3}$ exercised by the fiber, the fiber is strained in the radial direction by $\epsilon_{1}$ $=-\epsilon_{3 / 2}$.

0146-9592/80/030087-03\$0.50/0
Our next task is to determine the total phase retardation of a light beam propagating in the fiber caused by the strain induced by $H_{1}$. Using the form of the photoelastic tensor $p_{i k}$,

$$
\Delta\left[\frac{1}{n^{2}}\right]_{i}=p_{i k l} \epsilon_{k l} \quad(i=1,2, \ldots, 6),
$$

in an isotropic medium, ${ }^{2}$ and putting $\epsilon_{1}=\epsilon_{2}, \epsilon_{4}=\epsilon_{6}=$ 0 and replacing $k l$ by its contraction, we have

$$
\Delta\left[\frac{1}{n^{2}}\right]_{1}=\left(p_{11}+p_{12}\right) \epsilon_{1}+p_{12} \epsilon_{3}
$$

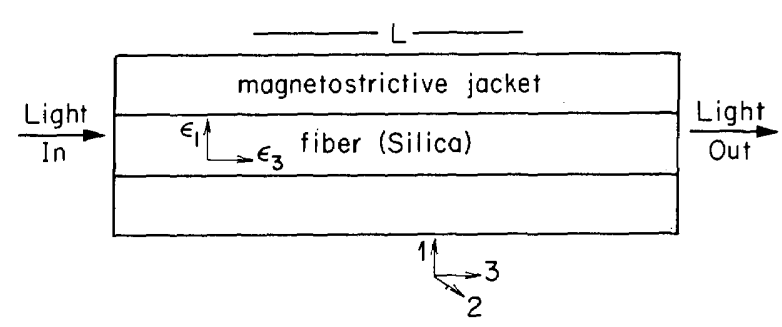

Fig. 1. The basic geometry of a silica fiber embedded in magnetostrictive jacket.

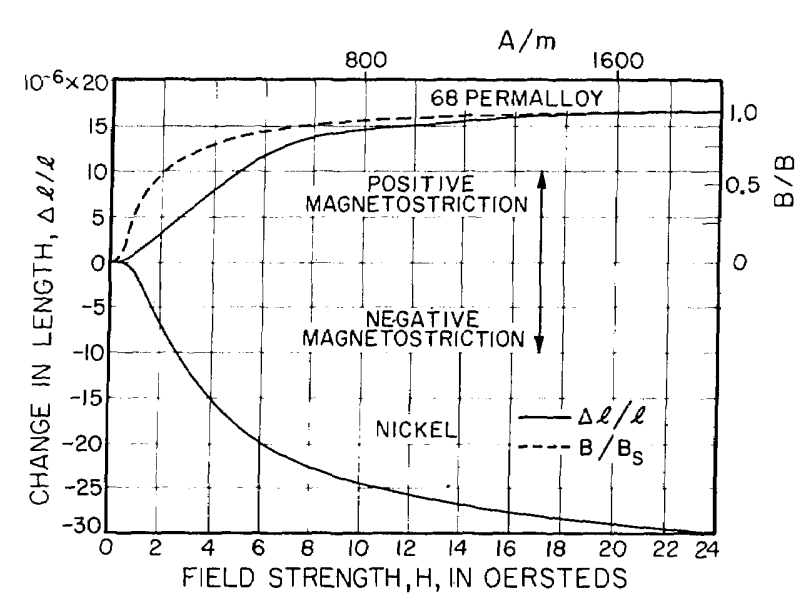

Fig. 2. The magnetostriction of nickel and of 68 permalloy. Data taken from Ref. 1.

(C) 1980, Optical Society of America 


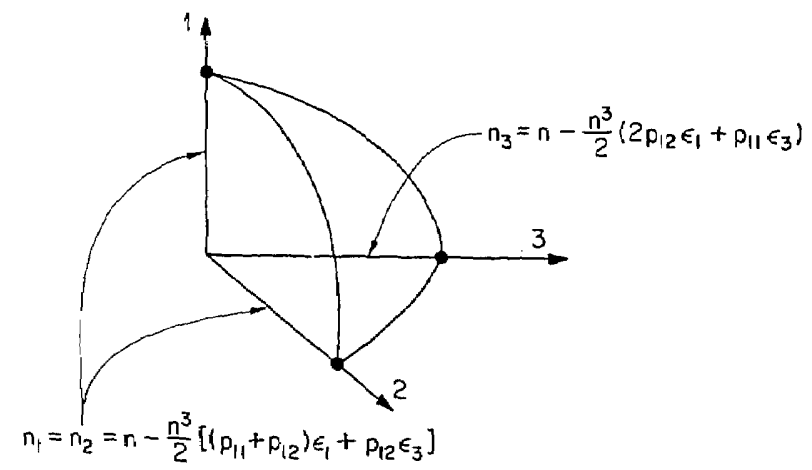

Fig. 3. The effect of applying strains $\epsilon_{1}$ and $\epsilon_{3}$ on the indicatrix of an isotropic material.

$$
\begin{aligned}
& \Delta\left[\frac{1}{n^{2}}\right]_{2}=\left(p_{11}+p_{12}\right)_{1}+p_{12} \epsilon_{3} \\
& \Delta\left[\frac{1}{n^{2}}\right]_{3}=2 p_{12} \epsilon_{1}+p_{11} \epsilon_{3} .
\end{aligned}
$$

Here $\left[1 / n^{2}\right]_{i}$ are the constants of the optical indicatrix. ${ }^{3}$ According to Eq. (7), the effect of strains $\epsilon_{1}$ and $\epsilon_{3}$ in an isotropic medium is merely to change the magnitude of the principal dielectric axes without changing their direction. This is shown in Fig. 3.

Light propagating in the fiber is polarized along the transverse directions and thus will have its indices of refraction modified according to

$$
\Delta n_{1}=\Delta n_{2}=-\frac{n^{3}}{2}\left[\left(p_{11}+p_{12}\right) \epsilon_{1}+p_{12} \epsilon_{3}\right] .
$$

The total phase change of fight propagating in the fiber is the sum of a change that is due to $\Delta n$ as well as that due to physical elongation. Neglecting modal dispersion, the phase change in a length $L$ of fiber is

$$
\begin{aligned}
\Delta \phi \simeq \frac{\omega}{c} \Delta(n L) & =\frac{2 \pi L}{\lambda}\left(\frac{\Delta L}{L}+\frac{\Delta n}{n}\right) \\
& =\frac{2 \pi n L}{\lambda}\left\{\epsilon_{3}-\frac{n^{2}}{2}\left[\left(p_{11}+p_{12}\right) \epsilon_{1}\right.\right. \\
& \left.\left.+p_{12} \epsilon_{3}\right]\right\},
\end{aligned}
$$

where $\Delta L / L=\epsilon_{3}$. Using the data for fused quartz, $p_{11}$ $=0.12, p_{12}=0.27, n=1.46$, as well as $\epsilon_{1}=-\epsilon_{3 / 2}$ [see Eq. (5)], we obtain

$$
\Delta \phi=\frac{2 \pi n L}{\lambda} 0.92 \epsilon_{3}
$$

We note that the photoelastic effects that are due to $\epsilon_{1}$ and $\epsilon_{3}$ nearly cancel each other, so the main contribution to Eq. (8) is due to the physical elongation of the fiber. Last, we use Eq. (4) to relate the strain $\epsilon_{3}$ to the field $H_{1}$ to be detected. The result is

$$
\Delta \phi(\mathrm{rad})=-2.44 \times 10^{-5}\left(\frac{L}{\lambda}\right) H_{1}(\mathrm{G}),
$$

which, for $\lambda=1 \mu \mathrm{m}\left(10^{-6} \mathrm{~m}\right)$, becomes

$$
\Delta \phi(\mathrm{rad})=-2.44 \times 10^{1} H_{1}(\mathrm{G}) L(\mathrm{~m}) .
$$

This is a substantial effect. To appreciate the orders of magnitude involved, let us assume that we want to detect a field $H_{1}=10^{-5} \mathrm{G}$. This will give rise according to Eq. (10) to $\Delta \phi \sim 2.44 \times 10^{-1} \mathrm{rad} \simeq 14^{\circ}$, if $L=10^{3} \mathrm{~m}$ $(1 \mathrm{~km})$. This is a very large phase shift and is easily detectable. A convenient detection scheme will be to mix the light beam at the output of the magnetostrictive fiber with that of a second reference fiber, which is not sensitive to the magnetic field, in a standard interferometric system. If the phase delay at the detector of the output fields from the fibers is $\pi / 2$ (this constant phase delay will be maintained by a separate feedback loop), then the signal output current from the mixing detector will be

$$
\begin{aligned}
i_{\mathrm{s}} & \simeq \frac{P e \eta}{h \nu} \Delta \phi \\
& \simeq \frac{P e \eta}{h \nu} 2.44 \times 10^{1} L H_{1} .
\end{aligned}
$$

In Eq. (11), $P$ is the total power in the optical beam, $e$ is the electron charge, and $\eta$ is the quantum efficiency of the detector. If $H_{1}$ is time varying, this time dependence will be imparted to the current $i_{s}$. The magnetic field $H_{0}$ is used to bias the magnetostrictive material to the point of maximum sensitivity. From Fig. 2 we see that for $\mathrm{Ni}$ this takes place for $\mathrm{H}_{0} \simeq 3 \mathrm{G}$. Such a low-bias field can be provided by coiling a current-carrying wire along the fiber or by placing the coiled fiber in a very weak electromagnet or permanent magnet.

The above calculations are just meant to illustrate the potential attractiveness of a fiber magnetostrictive magnetometer. An interesting effect to investigate will be that of magnetic-domain snapping on the device sensitivity (we expect this effect to become less important in long fibers because of averaging over many domains).

If we ignore the domain noise mechanism, then the most important source of noise is likely to be the shot noise

$$
\overline{i_{n}^{2}}=2 e\left(\frac{P e \eta}{h \nu}\right) \Delta \nu
$$

associated with the average current output from the mixing detector. The signal-to-noise power ratio is obtained from Eqs. (11) and (12) as

$$
\begin{aligned}
\frac{S}{N} & =\frac{\overline{i_{s}^{2}}}{\overline{i_{N}^{2}}}=\frac{P \eta(\Delta \phi)^{2}}{4 h \nu \Delta \nu} \\
& =\frac{148.8 P \eta L^{2} H_{i}^{2}}{h \nu \Delta \nu} .
\end{aligned}
$$

The minimum detectable $H_{1}$ is defined as that value of $H_{1}$ for which $S / N=1$. From Eq. (13),

$$
\left(H_{1}\right)_{\text {min shot noise }}=\sqrt{\frac{6.7 \times 10^{-3} h \nu \Delta \nu}{P_{\eta} L^{2}}} .
$$

Using a detection bandwidth $\Delta \nu=1 \mathrm{~Hz}, P=10^{-3} \mathrm{~W}$, $L=10^{3} \mathrm{~m}, \eta=0.5$, and $\nu=3 \times 10^{14} \mathrm{~Hz}(\lambda=1 \mu \mathrm{m})$, we obtain

$$
\left(H_{1}\right)_{\min } \simeq 1.6 \times 10^{-12} \mathrm{G} .
$$

Other real-life noise mechanisms will undoubtedly be found, but we will not speculate on their origins at this point. 
Before concluding this analysis, it may be interesting to compare the scheme considered here with that of detecting magnetic fields by Faraday rotation in fibers. 4,5 This rotation is due to paramagnetic impurities. The amount of such rotation is given by

$$
\theta=V H L,
$$

where $V$ is the Verdet constant, $L$ is the length of the fiber, and $H$ is the magnetic field. The maximum useful length of fiber is $\sim \alpha^{-1}$, where $\alpha$ is the absorption coefficient at the wavelength of the light used. The maximum rotation is then

$$
\theta_{\max } \simeq \frac{V H}{\alpha},
$$

which, for typical good glasses, is $\sim 6 \mathrm{~min} / \mathrm{G}$. By way of comparison, the magnetostrictive effect yields [see Eq. (10)] $\Delta \phi \sim 2.44 \times 10^{4} \mathrm{rad} / \mathrm{G}$ in $1 \mathrm{~km}$ of fiber. Faraday rotation is thus a considerably smaller effect.
The authors are indebted to Douglas Pinnow, Jim Rice, and Tom McGill for useful discussions.

* Permanent address, Department of Applied Physics, 116-81, California Institute of Technology, Pasadena, California 91125.

$\dagger$ Permanent address, Defense Advanced Research Projects Agency, 1400 Wilson Boulevard, Arlington, Virginia 22209.

\section{References}

1. F. M. Bozorth, Ferromagnetism (Van Nostrand, New York, 1951).

2. J. F. Nye, Physical Properties of Material (Oxford U. Press, Oxford, England, 1957).

3. A. Yariv, Quantum Electronics, 2nd ed. (Wiley, New York, 1975).

4. A. Papp and K. Kempter, Appl. Opt. 15, 799 (1976).

5. S. C. Rasleign and R. Ulrich, Appl. Phys. Lett. 34, 768 (1979). 